\title{
The Evolution and Space Density of Damped Lyman- $\alpha$ Galaxies
}

\author{
Céline Péroux 7 , Mike J. Irwin and Richard G. McMahon \\ Institute of Astronomy, University of Cambridge, UK \\ Lisa J. Storrie-Lombardi \\ SIRTF Science Center, California Institute of Technology, Pasadena, USA
}

\begin{abstract}
The results of a new spectroscopic survey of $66 z \gtrsim 4$ quasars for Damped Lyman- $\alpha$ absorption systems are presented. The search led to the discovery of 30 new DLA candidates which are analysed in order to compute the comoving mass density of neutral gas in a non-zero $\Lambda$ Universe. The possible sources of uncertainty are discussed and the implications of our results for the theories of galaxy formation and evolution are emphasized. A subsequent paper will present details of the calculations summarised here and a more extensive explanation of the consequences of our observations for the understanding of the nature of DLAs.
\end{abstract}

\section{A New Survey for Damped Lyman- $\alpha$ Galaxies}

Damped Lyman- $\alpha$ (hereafter DLA) galaxies are defined as absorption systems in the spectra of quasars with a hydrogen column density $N(H I)>2 \times 10^{20}$ atoms $\mathrm{cm}^{-2}(\geq 10 \AA$ rest frame equivalent width, Wolfe et al. 1986). Their space density distribution, metal abundances and kinematics seem to indicate that they are the progenitors of present day galaxies, thus providing a powerful observational tool for galaxy evolution studies.

DLAs probe the neutral gas from which stars form and thus are a direct means to trace galaxy formation. Quasars are extremely luminous objects which are observed up to $z=5.8$ (Fan et al. 2000), and thus DLAs can be probed over a large redshift range. Finally DLA galaxies can be detected to fainter limits than objects detected using traditional emission observations.

We observed $66 z \gtrsim 4$ quasars (see Peroux et al. 2000 for details on the observations) from the $2^{\text {nd }}$ APM colour survey (Storrie-Lombardi et al. 2000), the Palomar Sky Survey (Kennefick et al. 1995a \& 1995b and G. Djorgovski's web page) and the Sloan Digital Sky Survey (Fan et al. 1999). This resulted in 31 DLA candidates, 30 of which are newly discovered and many with associated metal lines.

\footnotetext{
*e-mail: celine@ast.cam.ac.uk
} 


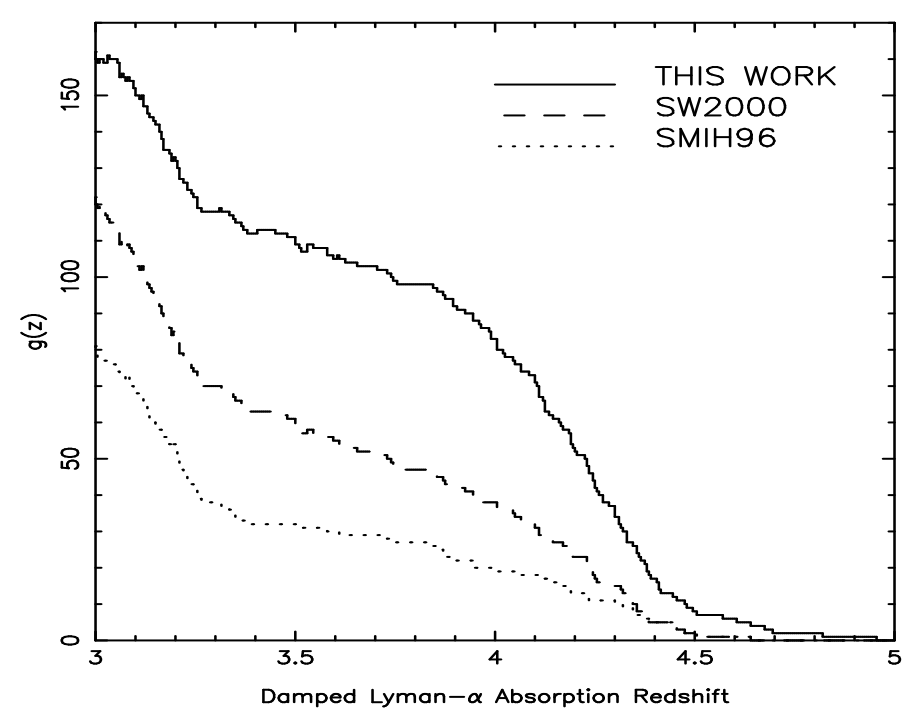

Figure 1. Sensitivity functions for DLA galaxy searches. The dotted line is from Storrie-Lombardi et al. $1996(\Delta(z)=239)$, the dashed line is the compilation of Storrie-Lombardi \& Wolfe $2000(\Delta(z)=418)$, and our survey is represented by the solid line $(\Delta(z)=493)$. Our new sample more than doubles the redshift path surveyed above $z \gtrsim 3.5$.

\section{Probing the Neutral Gas Content of the Universe}

For each quasar the redshift range along which a DLA could be observed is determined and used to deduce the survey's sensitivity. This is represented by the $g(z)$ function shown in Fig 1 . Our new data is analysed in conjunction with previous observations resulting in 115 DLAs observed in 697 quasars (Rao and Turnshek 1999 data not included).

The mass of neutral gas contained in DLAs is expressed as: $\Omega_{D L A}(z)=$ $\frac{H_{o} \mu m_{H}}{c \rho_{c r i t}} \int_{N_{m i n}}^{\infty} N f(N, z) d N$, where $N$ is the column density and $f(N, z)$ is the distribution function. The integral is estimated by summing up over all the observed column densities and dividing by the absorption distance interval, $X(z)$, which corrects for comoving coordinates and thus depends on the geometry of the Universe. In a non-zero $\Lambda$ Universe: $X(z)=\int_{0}^{z}(1+z)^{2}\left[(1+z)^{2}\left(1+z \Omega_{M}\right)-z(2+z) \Omega_{\Lambda}\right]^{-1 / 2} d z$.

Our observations are presented in Fig 2 for a currently popular cosmology and show the evolution of $\Omega_{D L A}$ over the last $90 \%$ of the age of the Universe thus probing the early stages of galaxies even before gas turns into stars.

In order to account for the neutral gas not included in DLAs, we used a power law with an exponential turnover (similar to the Schechter function) in extrapolating from the observed column density distribution: 


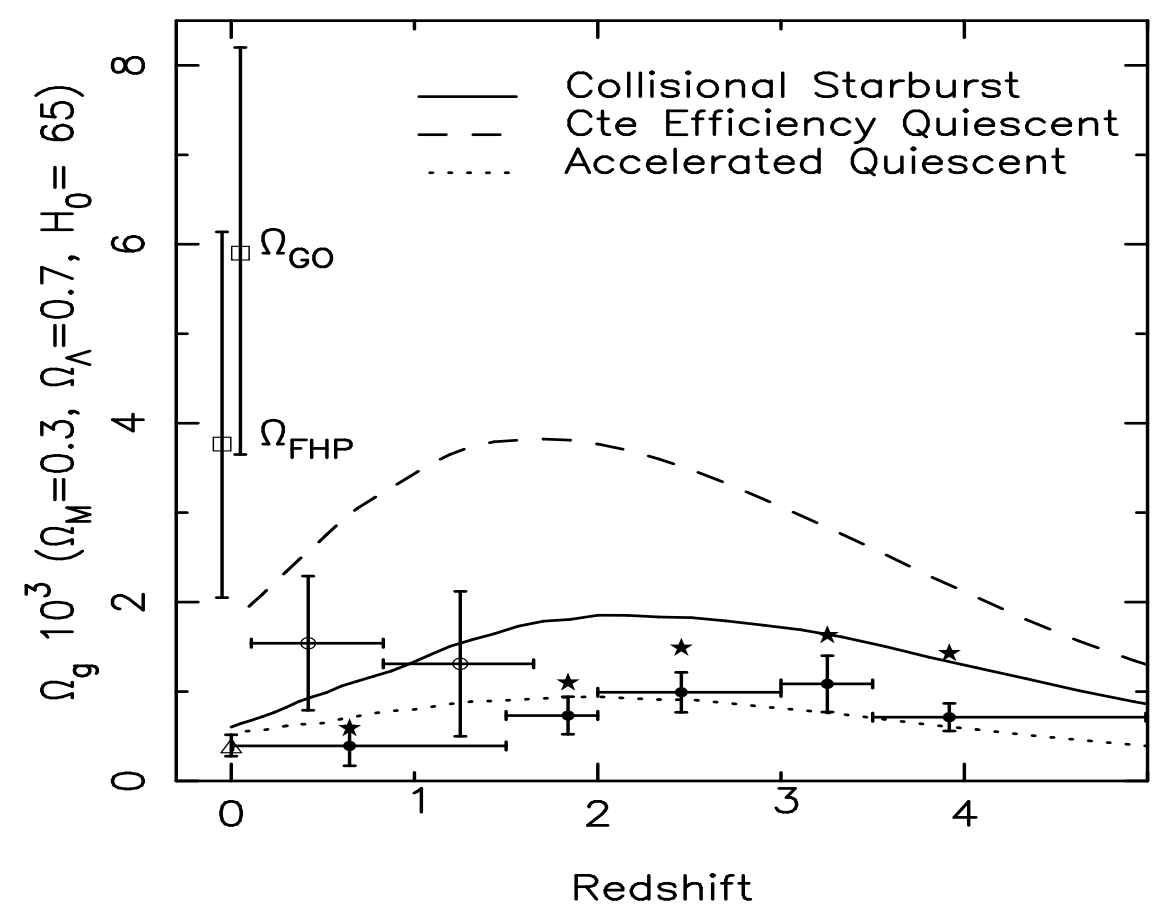

Figure 2. The circles show the neutral gas in Damped Lyman- $\alpha$ galaxies (the filled circles are our observations and the open circles are Rao \& Turnshek 1999 results). Vertical bars correspond to $1 \sigma$ errors and the horizontal bars indicate bin sizes. The stars are the total HI including a correction for the neutral gas contained in systems with column densities below $2 \times 10^{20}$ atoms $\mathrm{cm}^{-2}$. The triangle at $z=0$ is from Natarajan \& Pettini 1997 who used a recent galaxy luminosity function to reproduce the local HI mass measured by Rao \& Turnshek 1993. The squares, $\Omega_{F H P}$ and $\Omega_{G O}$ (Fukugita, Hogan \& Peebles 1998 and Gnedin \& Ostriker 1992 respectively) are $\Omega_{\text {baryons }}$ in local galaxies. The semi-analytical models from Somerville, Primack \& Faber 2000 are overplotted (see text for detailed explanations of the models).

$f(N, z)=\left(f_{*} / N_{*}\right)\left(N / N_{*}\right)^{-\beta} e^{-N / N_{*}}$. Using the parameters determined by Storrie-Lombardi et al. 1996, $N_{*}=21.63 \pm 0.35$ and $\beta=1.48 \pm 0.30$, results in a $\sim 20 \%$ increase in $\Omega$ for $z \leq 3.5$. For $z>3.5$ systems, our observations indicate a steepening of the low column density end of the DLA distribution (where much of the mass resides) in comparison with our previous study and leads to a factor of $\sim 2$ correction (as shown by the stars in Fig 2).

\section{Discussion}

Most of the uncertainties discussed here seem to indicate that $\Omega_{D L A}$ is being underestimated. At low redshift, Rao \& Turnshek 1999 used a 
different method (based on the observations of Lyman- $\alpha$ in identified Mg II systems) and HST data to derive a higher neutral gas content in DLAs (Fig 2). These results are surprisingly high and might be due to low number statistics or might indicate that high redshift results are underestimated. More uncertainties arise in the fact that dust is likely to produce observational biases unaccounted for here and that the exact nature of DLAs is unknown. In particular, they might be different types of object at different redshift. Finally, higher resolution data are necessary in order to identify multiple systems.

Several groups (Kauffmann \& Haehnelt 2000, Somerville, Primack \& Faber 2000) have included more realistic physics in their simulations to construct semi-analytical models of galaxy formation which, among other things, predict the evolution of cold gas in the Universe. The models presented in Fig 2 vary in their recipe for star formation: it is triggered by galaxy-galaxy mergers in the collisional starburst model, constant with redshift in the constant efficiency model, and scales inversely with disc dynamical time in the accelerated efficiency model. Our observational results can thus be used to directly constrain theories of galaxy evolution.

We would like to thank Isobel Hook for help in acquiring the data. $\mathrm{CP}$ is grateful to Max Pettini and the organisers for help with a very enjoyable conference.

\section{References}

Djorgovski's www page at http://astro.caltech.edu/ george/z4.qsos

Gnedin, N. \& Ostriker, J., 1992, ApJ, 400, 1.

Fan, X. et al. 2000, in press, astro-ph/0005414.

Fan, X. et al. 1999 ApJ, 118, 1.

Fukugita, M., Hogan, C. \& Peebles, P. 1998, ApJ, 503, 518.

Kauffmann, G. \& Haehnelt, M. 2000, MNRAS, 311, 576.

Kennefick, J., De Carvalho, R., Djorgovski, G., Wilber, M., Dickson, E., \& Weir, N. 1995, ApJ, 110, 78.

Kennefick, J., Djorgovski, S. \& De Carvalho, R. 1995, ApJ, 100, 2553.

Natarajan, P. \& Pettini, M. 1997, MNRAS, 291, L28.

Peroux, C., Storrie-Lombardi, L., McMahon, R. \& Irwin, M. 2000, in preparation.

Rao, S. \& Briggs, F. 1993, ApJ, 419, 515.

Rao, S. \& Turnshek, D. 2000, ApJS, in press, astro-ph/9909164.

Somerville, R., Primack, J. \& Faber, S. 2000, MNRAS, in press, astro-ph/0006364.

Storrie-Lombardi, L., McMahon, R., \& Irwin, M. 1996, MNRAS, 283, L79.

Storrie-Lombardi, L., Irwin, M., McMahon, R., \& Hook, I., 2000, in preparation.

Storrie-Lombardi, L. \& Wolfe, A. 2000, ApJ, in press, astro-ph/0006044.

Wolfe, A., Turnshek, D., Smith, H.\& Cohen, R. 1986, ApJS, 61, 249. 